\title{
Is NIRS monitoring well tolerated in term and preterm neonates?
}

\author{
EVELYN ZIEHENBERGER ${ }^{1,2}$, BERNDT URLESBERGER ${ }^{1,2}$, CORINNA BINDER-HESCHL ${ }^{1,2}$, BERNHARD \\ SCHWABERGER ${ }^{1,2}$, NICHOLAS MORRIS ${ }^{2}$, NARIAE BAIK ${ }^{1,2}$, ALEXANDER AVIAN ${ }^{3}$, GERHARD PICHLER ${ }^{1,2,4,5}$ \\ ${ }^{1}$ Research Unit for Cerebral Development and Oximetry Research, Division of Neonatology, Department of Paediatrics, Medical University of Graz, Austria \\ ${ }^{2}$ Division of Neonatology, Department of Paediatrics, Medical University, Graz, Austria \\ ${ }^{3}$ Institute for Medical Informatics, Statistics and Documentation, Medical University of Graz, Austria \\ ${ }^{4}$ Department of Paediatrics, University of Alberta, Edmonton, Canada \\ ${ }^{5}$ Neonatal Research Unit, Royal Alexandra Hospital, Edmonton, Canada
}

Corresponding autor:

Gerhard Pichler

Division of Neonatology, Department of Paediatrics

Medical University of Graz

Auenbruggerplatz 30, $8036 \mathrm{Graz}$, Austria

Phone: +4331638581133

Fax: +4331638512678

E-mail: pichler.gerhard@klinikum-graz.at

\section{ABSTRACT}

Background. Near infrared spectroscopy (NIRS) is a new, non-invasive monitoring method in neonates, which has now become part of routine monitoring in many neonatal intensive care units (NICU). New, additional, non-invasive technical monitoring might have an influence on neonatal wellbeing.

Objectives. The aim of the present study was to evaluate the wellbeing of neonates during peripheral and cerebral NIRS monitoring and venous occlusions.

Methods. In the present study, secondary outcome parameters of prospective observational studies with NIRS in term and preterm neonates were analysed. Heart rate (HR), arterial oxygen saturation ( $\mathrm{SpO} 2)$, respiratory rate $(\mathrm{RR})$, mean arterial blood pressure (MABP), pain score and skin condition at four defined time points during NIRS measurements of regional tissue oxygenation were recorded and analysed. Results. Thirty-six term and preterm neonates were included (gestational age (GA) $36 \pm 1$ week, 2779 $\pm 930 \mathrm{~g}$ birth weight). $\mathrm{SpO} 2$ and peripheral tissue oxygenation index (pTOI) increased and HR dropped while RR, mean MABP and pain scores remained unchanged during NIRS measurements. In eight infants, a mildly irritated area of skin was noted where the NIRS sensors had been attached.
Conclusion. Cerebral and peripheral NIRS monitoring and venous occlusions were painless and well tolerated by term and preterm neonates.

Key words: neonates, NIRS, vital signs

\section{INTRODUCTION}

In 1977 Jöbsis (1) described a new means of monitoring regional tissue oxygen saturation, with near infrared spectroscopy (NIRS), which has now become part of routine monitoring in many neonatal intensive care units. By applying sensors to the skin, NIRS is able to estimate regional oxygen saturation in various regions of interest (brain, muscle, kidney, liver etc.) noninvasively and continuously. (2) For cerebral NIRS measurements, sensors are often attached to the forehead, for peripheral measurements they are attached to the skin at the area of interest - e.g. abdominal skin closest to kidney or liver. For peripheral muscle measurements the sensors are attached on the skin of the lower or upper limb and measurements can be combined with the venous occlusion techniques. (35)

NIRS is non-invasive which makes it ideal for routine purposes in addition to arterial oxygen saturation ( $\mathrm{SpO} 2)$, electrocardiogram (HR), oscillometric blood pressure monitoring or temperature measurements. Depending on the type of NIRS measurement it can be attached to the skin by means of either a probe holder and an adhesive strip or an elastic bandage. During routine care the sensors can be attached securely to permit uncompromised positioning on the infants.

Every intervention in term and preterm neonates might cause discomfort and might not be well tolerated. Therefore, after introduction of new monitoring in neonates, it is necessary to evaluate tolerance and influence on well-being. Response to discomfort or pain can be evaluated in term and preterm neonates with scoring systems that include different items, such as facial expression, changes in motor behaviour and alterations in vital parameters. $(6,7)$

The aim of this study was to evaluate the impact of peripheral and cerebral NIRS monitoring and peripheral occlusions on vital parameters, pain score and skin integrity and to make a judgement on tolerance of these measurements. (8) For this purpose we recorded vital parameters, pain scores, skin condition and duration of manipulation. We hypothesised that NIRS measurements are well tolerated with no influence on vital parameters, pain score and skin integrity. 


\section{METHODS}

In a prospective observational study with NIRS (EK.NR: 21-149 ex 09/10), we included term and preterm neonates who were admitted to our neonatal intensive care unit. Written consent of parents was obtained prior to inclusion in the study.

Standard monitoring during NIRS measurements consisted of HR with electrocardiogram, SpO2 with pulse-oximetry attached to the left foot, respiratory rate (RR), and a blood pressure measurement with the cuff applied to the left thigh (Philips Inteli View MP50 Netherland). NIRS measurements were performed using the NIRO 300 (Hamamatsu Photonics, Japan). The sensor for cerebral measurements (cTOI- cerebral tissue oxygenation index) was attached to the forehead using elastic bandaging and probe holder. The sensor for peripheral muscle measurement (pTOI- peripheral tissue oxygenation index) was attached to the left calf using a probe holder, adhesive strip and adhesive bandage. The temperature probe was fixed next to pTOI. In all infants both peripheral and cerebral NIRS measurements were done simultaneously. NIRS measurements of pTOI followed a standardised protocol using intermittent inflations of a blood pressure cuff causing venous occlusion in the left thigh still permitting arterial perfusion. (3-5) After the attachment of sensors and after the infant was settled three consecutive venous occlusions were performed with rest periods of one minute between occlusions. The blood pressure cuff was inflated for 20 seconds to a pressure of between 20 and $40 \mathrm{mmHg}$ for venous occlusions.
We defined four time points for measurements of vital parameters and pain score: before manipulation of the infant, after attachment of sensors, before the first venous occlusion and after measurement and removal of sensors. At each time point we recorded $\mathrm{HR}, \mathrm{SpO} 2, \mathrm{RR}$ and mean arterial blood pressure (MABP) as routine parameters and in addition cTOI and pTOI with NIRS. The sensors were removed using Dermasol ${ }^{\bullet}$. We checked the skin once before and once after NIRS measurements and classified the local skin as not irritated, mildly irritated, moderately irritated or as loss of integrity. Central and peripheral temperature was measured before manipulation and after the fourth time point.

In infants without respiratory support scoring was done using the KUSS scoring system which is an infant discomfort and pain score. (9) We used the KUSS score to quantify discomfort and pain, because it is validated for infants from birth to the age of 4 years, and because it as a score, which is validated for the German language. It looks at infant behaviour and movement by evaluating 5 items - crying, facial expression, posture of trunk, posture of legs, and hyperactivity. Behaviour is scored between zero and two points, thus showing a maximum scoring of ten points, and a minimum of zero points. (9) A value of four points is considered as cut off value for start of pharmacological pain therapy. The scoring was done before manipulation and after measurement in $15 \mathrm{sec}$.

Vital parameters and KUSS score at time points two, three and four were compared to readings at time point one by means of
ANOVA for repeated measurements or Friedman's Test. Post hoc tests for analysing changes from time point 1 to all other time points were done using paired t-Test or Wilcoxon signed-rank test. Regional cerebral and peripheral oxygenation at time points three and four were compared to readings at time point two by means of ANOVA for repeated measurements. Post hoc tests to analyse changes from time point 2 to all other time points were done using paired t-Test.

\section{RESULTS}

From August 2010 until July 2011 we were able to include 36 term and preterm infants in our study. Demographic data are presented in table 1. Duration of NIRS measurements was $61 \pm 17$ minutes (mean \pm SD). None of the infants experienced respiratory or circulatory problems or were treated with sedatives during measurements. HR at time point 2, 3 and 4 was significantly lower compared to $\mathrm{HR}$ at time point 1 . SpO2 at time point 2 and 3 was significantly higher than at time point 1 (table 2). No change of RR, MABP and of KUSS score was observed at different time points. cTOI levels were stable throughout measurements, whereas pTOI was significantly higher at time point 3 compared to time point 2 (table 3 ). There were no skin irritations on the forehead after measurements, whereas eight infants showed mildly irritated skin after removal of sensors from the lower limb.

Table 1. Demographic data for 36 term and preterm neonates.

\begin{tabular}{ll}
\hline Gestational age (week) & $36.1 \pm 1$ \\
\hline Postnatal age $(\mathrm{d})$ & $2 \pm 1$ \\
\hline Birth weight $(\mathrm{g})$ & $2779 \pm 930$ \\
\hline Actual weight $(\mathrm{g})$ & $2747 \pm 912$ \\
\hline Temperature, peripheral $\left({ }^{\circ} \mathrm{C}\right)$ & $35 \pm 0.5$ \\
\hline Temperature, rectal $\left({ }^{\circ} \mathrm{C}\right)$ & $37 \pm 0.3$ \\
\hline Average duration $(\min )$. & $61 \pm 17$ \\
\hline
\end{tabular}

mean score \pm standard deviation 
Table 2. Vital parameters and KUSS score during the near infrared spectroscopy (NIRS) measurements in 36 term and preterm neonates.

\begin{tabular}{|c|c|c|c|c|}
\hline & Before manipulation & After attachment & Before occlusion & After measurement \\
\hline $\begin{array}{l}\text { Heart rate }(\mathrm{bpm}) \\
(\mathrm{n}=33)\end{array}$ & $133 \pm 14$ & $\begin{array}{l}127 \pm 14^{*} \\
\mathrm{p}=.002 \\
\mathrm{~T}=3.39\end{array}$ & $\begin{array}{l}128 \pm 16^{*} \\
\mathrm{p}=.020 \\
\mathrm{~T}=2.46\end{array}$ & $\begin{array}{l}127 \pm 12^{*} \\
\mathrm{p}<.001 \\
\mathrm{~T}=3.99\end{array}$ \\
\hline $\begin{array}{l}\text { Arterial oxygen saturation }(\%) \\
(\mathrm{n}=33)\end{array}$ & $96 \pm 3$ & $\begin{array}{l}98 \pm 2^{*} \\
\mathrm{p}=.001 \\
\mathrm{~T}=-3.84\end{array}$ & $\begin{array}{l}97 \pm 2^{*} \\
\mathrm{p}=.031 \\
\mathrm{~T}=-2.25\end{array}$ & $\begin{array}{l}97 \pm 3 \\
\mathrm{p}=.225 \\
\mathrm{~T}=-1.24\end{array}$ \\
\hline $\begin{array}{l}\text { Respiratory rate (breath/min.) } \\
(\mathrm{n}=33)\end{array}$ & $51 \pm 17$ & $\begin{array}{l}54 \pm 15 \\
\mathrm{p}=.432 \\
\mathrm{~T}=-0.80\end{array}$ & $\begin{array}{l}51 \pm 15 \\
\mathrm{p}=.957 \\
\mathrm{~T}=0.05\end{array}$ & $\begin{array}{l}48 \pm 14 \\
\mathrm{p}=.362 \\
\mathrm{~T}=0.93\end{array}$ \\
\hline $\begin{array}{l}\text { Mean arterial blood pressure }(\mathrm{mmHg}) \\
(\mathrm{n}=23)\end{array}$ & $42 \pm 7$ & $\begin{array}{l}45 \pm 9 \\
\mathrm{p}=.156 \\
\mathrm{~T}=-1.47\end{array}$ & $\begin{array}{l}43 \pm 9 \\
\mathrm{p}=.552 \\
\mathrm{~T}=-0.60\end{array}$ & $\begin{array}{l}45 \pm 7 \\
\mathrm{p}=.188 \\
\mathrm{~T}=-1.36\end{array}$ \\
\hline $\begin{array}{l}\text { KUSS score } \\
\text { (max. } 10 \text { points) }\end{array}$ & $0(0-2)$ & $\begin{array}{l}0(0-2) \\
p=.317 \\
Z=-1.00\end{array}$ & $\begin{array}{l}0(0-2) \\
p=.564 \\
Z=-0.58\end{array}$ & $\begin{array}{l}0 \\
\mathrm{p}=.317 \\
Z=-1.00\end{array}$ \\
\hline
\end{tabular}

mean \pm standard deviation, median(range); ${ }^{\star}$ p-value $<0.05$ for comparison to time point "Before manipulation", p-values, T-values for paired t-Test and $\mathrm{Z}$-values for Wilcoxon signed-rank test are given.

Table 3. Near infrared spectroscopy (NIRS) parameters for 36 term and preterm neonates.

\begin{tabular}{|c|c|c|c|}
\hline & After attachment & Before occlusion & After measurement \\
\hline Cerebral tissue oxygenation index & $71 \pm 13$ & $73 \pm 7$ & $73 \pm 7$ \\
\hline (\%) & & $\mathrm{p}=.610$ & $\mathrm{p}=.526$ \\
\hline$(\mathrm{n}=30)$ & & $\mathrm{T}=-0.52$ & $\mathrm{~T}=-0.64$ \\
\hline Peripheral tissue oxygenation & $69 \pm 6$ & $72 \pm 6^{*}$ & $71 \pm 5$ \\
\hline index $(\%)$ & & $\mathrm{p}=.009$ & $\mathrm{p}=.064$ \\
\hline$(n=33)$ & & $\mathrm{T}=-2.79$ & $\mathrm{~T}=-1.92$ \\
\hline
\end{tabular}

mean \pm standard deviation; ${ }^{\star} \mathrm{p}$-value $<0.05$ for comparison to time point "After attachment", $\mathrm{p}$-values and T-values for paired t-Test are given.

\section{DISCUSSION}

Based on the evaluation of vital parameters, pain scores and clinical observation in the present study, peripheral or cerebral NIRS measurements do not pose a significant burden on the wellbeing of term and preterm neonates.

According to Gonsalves and Mercer (7), manipulations causing any stress would result in an opposite behaviour of HR and $\mathrm{SpO} 2$. They compared vital parameters in preterm infants undergoing painful procedures (injections, heel stick, tape removal) and non-painful procedures (handling, temperature taking, alcohol swabbing, feeding) and found an increase in HR and $\mathrm{RR}$ and a decrease in SpO2 in the painful procedure group. Our observation of reduction in $\mathrm{HR}$ and increase in $\mathrm{SpO} 2$ is in accordance with non-painful procedures. The cTOI remained unchanged and pTOI increased slightly during the observation period. Catelin et al. (6) showed that cTOI itself may serve as a useful parameter to capture painful and stressful procedures in neonates that might show a decrease and an increase during behavioural interventions. The lack of changes of cTOI in the present study further support the tolerability of NIRS measurements and venous occlusions.

In our study, using the KUSS score, we found no evidence of pain or discomfort brought upon infants by the NIRS measurements. There are three categories of negative experiences for a newborn: pain, discomfort and general disturbances. (10) Important behavioural changes caused by pain in non-ventilated and non-sedated newborns are well captured by the KUSS score. NIRS is a non-invasive tool and therefore can be seen as non-painful. The KUSS score should have also captured any form of discomfort associated with NIRS measurements. However, additional dis- turbances caused by manipulations associated with NIRS measurements could be relevant. Integration of manipulation due to NIRS measurements into routine nursing handling schemes, might overcome an increased burden due to manipulation.

In regards to skin condition and integrity, with a mean measurement time of $61 \mathrm{~min}$ utes, we only evaluated short-term use. There were no skin irritations caused by cerebral measurements, but we observed signs of mild irritation in peripheral measurements. No severe adverse reactions associated with long term cerebral NIRS measurements have been recorded, but skin marks have been reported in a larger randomized trial. (11) For the long-term use of NIRS, re-positioning of the sensor seems therefore to be appropriate to avoid problems.

In conclusion, cerebral and peripheral NIRS monitoring and venous occlusions 
can be considered a painless, well tolerated, non-invasive method for monitoring cerebral and peripheral tissue oxygenation and haemodynamic status, which can in addition be well integrated into routine neonatal management.

\section{REFERENCE}

1. Jöbsis FF. Noninvasive, infrared monitoring of cerebral and myocardial oxygen sufficiency and circulatory parameters. Science 1977;198(4323):1264-7.

2. Nicklin SE, Hassan IA-A, Wickramasinghe YA, Spencer SA. The light still shines, but not that brightly? The current status of perinatal near infrared spectroscopy. Arch Dis Child Fetal Neonatal Ed 2003;88(4):F263-8.

3. Grossauer K, Pichler G, Schmoelzer G, Zotter H, Mueller W, Urlesberger B. Comparison of peripheral and cerebral tissue oxygenation index in neonates. Arch Dis Child Fetal Neonatal Ed 2009;94(2):F15. doi:10.1136/adc.2008.146654.

4. Pichler G, Heinzinger J, Kutschera J, Zotter H, Müller W, Urlesberger B. Forearm and Calf Tissue Oxygenation in Term Neonates Measured with Near-Infrared. J Physiol Sci 2007;57 (5):317-9. doi:10.2170/physiolsci.SC004407.

5. Pocivalnik M, Pichler G, Zotter H, Tax N, Müller W, Urlesberger B. Regional tissue oxygen saturation: comparability and reproducibility of different devices. J Biomed Opt 2011;16(5):57004. doi:10.1117/1.3575647.

6. Catelin C, Tordjman S, Morin Vincen, Oger E, Sizun J. Clinical, Physiologic, and Biologic Impact of Environmental and Behavioral Interventions in Neonates During a Routine Nursing Procedure. J Pain 2005;6(12):791-7. doi:10.1016/j.jpain.2005.07.010

7. Gonsalves S, Mercer J. Physiological correlates of painful stimulation in preterm infants. Clin J Pain 1993;9(2):88-93.

8. Murdoch DR and Darlow BA. Handling during neonatal intensive care. Arch Dis Childhood 1984;59:957-61.

9. Büttner W, Finke W, Hilleke M, Reckert S, Vsianska L, Brambrink A. Development of an observational scale for assessment of postoperative pain in infants. Anästhesiol Intensivmed 1998;33(06):353-61. doi:10.1055/s-2007-994263.

10. VandenBerg KA. Individualized developmental care for high risk newborns in the NICU: A practice guidline. Elsevier Ireland Ltd. 2007. doi:10.1016/j.earlhumdev.2007.03.008

11. Hyttel-Sorensen S1, Austin T, van Bel F, Benders M, Claris O, Dempsey E, et al. A phase II randomized clinical trial on cerebral near-infrared spectroscopy plus a treatment guideline versus treatment as usual for extremely preterm infants during the first three days of life (SafeBoosC): study protocol for a randomized controlled trial. Trials 2013 May 1;14:120. doi:10.1186/1745-6215-14-120. 